\title{
Non-resonant operation of microcavity Brillouin lasers
}

Dmitry A. Korobko, Igor O. Zolotovskii, Sergei G. Sokolovskii, Andrei A. Fotiadi

Dmitry A. Korobko, Igor O. Zolotovskii, Sergei G. Sokolovskii, Andrei A. Fotiadi, "Non-resonant operation of microcavity Brillouin lasers," Proc. SPIE 10904, Laser Resonators, Microresonators, and Beam Control XXI, $109041 \mathrm{U}$ (21 March 2019); doi: 10.1117/12.2508345

SPE. Event: SPIE LASE, 2019, San Francisco, California, United States 


\title{
Non-resonant operation of microcavity Brillouin lasers
}

\author{
Dmitry A. Korobko ${ }^{*}$, Igor O. Zolotovskii ${ }^{a}$, S.G. Sokolovski ${ }^{b}$, and Andrei A. Fotiadi ${ }^{\text {a b, c }}$ \\ ${ }^{a}$ Ulyanovsk State University, 42 Leo Tolstoy Street, Ulyanovsk, 432970, Russian Federation \\ ${ }^{\mathrm{b}}$ School of Engineering and Applied Science, Aston University, Birmingham, B4 7ET, UK \\ ${ }^{c}$ University of Mons, blvd. Dolez 31, Mons, B-7000, Belgium
}

\begin{abstract}
We present theoretical framework to describe Brillouin lasing in microcavities in the case of a significant mismatch between the Brillouin shift and the cavity intermode spacing. We show that despite an increase of the lasing threshold a significant increase of the Brillouin power in comparison with the resonance case is achievable. A necessary condition for this effect is the optimal value of the pump frequency detuning from the cavity mode frequency. An increase of the Brillouin threshold is accompanied by narrowing of the spectrum range where the Brillouin signal could be generated in non-resonant case. Besides, with the optimal pump frequency detuning the Brillouin signal noise level is reduced. Analytical results are in quantitative agreement with the results of numerical simulations.
\end{abstract}

Keywords: Stimulated Brillouin scattering; microresonators; fiber Brillouin laser

\section{INTRODUCTION}

Design of low noise narrow-band laser sources is one of tasks the modern photonics focuses on [1-3]. The scope of such laser applications is spread on distributed fiber monitoring [4, 5], precise spectroscopy [6], optical communications [7, 8], microwave photonics [9], and so on. The most accessible and studied method to narrow the laser linewidth is to organize a feedback loop to the laser generation $[10,11]$, namely a feedback through an external cavity [12-14]. The use of such configurations allows to narrow the linewidth of standard semiconductor DFB lasers down to several $\mathrm{kHz}$ [15]. Alternative solutions for design of narrow-band lasers are based on stimulated Brillouin scattering (SBS). They exploit specific properties of the Brillouin scattering amplification process such as a low-power threshold and a narrow gain band (tens of MHz) [16]. Such a narrow Brillouin gain spectrum that could be narrower than the cavity inter-mode spacing supports single-frequency Brillouin laser operation in cavities of lengths less than $\sim 10 \mathrm{~m}[17,18]$. Progress in high-Q micro-cavities based on classical optical materials (whispering gallery modes in quartz spheres, disks, etc. $[19,20]$ ) or based on chalcogenide glasses, silicon and other materials with an increased Brillouin gain factor [21-23] allows to demonstrate Brillouin lasers with extraordinary performance characteristics in terms of laser linewidth (down to hundreds of $\mathrm{Hz}$ or less) and low noise.

Effective SBS generation in microcavities requires adjusting of the cavity inter-mode spacing to the Brillouin frequency shift. The current advanced technology allows to control the microcavity size adjusting the cavity free spectrum range (FSR) with an accuracy of about $1 \mathrm{MHz}$, while its absolute value is comparable with the Brillouin frequency shift (typically, $10 \mathrm{GHz}$ ) [24]. However, the effects caused by the detuning of the FSR from the Brillouin shift on Brillouin lasing should be mandatorily accounted by the laser designers. Indeed, both parameters (FSR and $\Omega_{B}$ ) depend on the pump wavelength and external factors (temperature, strain, etc.). Besides, these effects could be used for the laser frequency tuning, the Brillouin frequency combs adjustment [25] or sensing of external perturbations [26].

Laser Resonators, Microresonators, and Beam Control XXI, edited by Alexis V. Kudryashov,

Alan H. Paxton, Vladimir S. Ilchenko, Proc. of SPIE Vol. 10904, 109041U · @ 2019 SPIE

CCC code: $0277-786 X / 19 / \$ 18 \cdot$ doi: $10.1117 / 12.2508345$ 
The aim of this work is to describe the dynamical effects arising in the Brillouin laser microcavities in the case of mismatching between the Brillouin frequency shift and the cavity FSR. Formally, the used approaches are valid for any type of Brillouin lasers with a cavity shorter than one meter, i.e. when a single-mode laser operation is supported by the Brillouin gain. Under these conditions, the frequency detuning $\left|\Omega_{B} / 2 \pi-k \mathrm{FSR}\right|$ is strongly less than tens of $\mathrm{MHz}$ excluding any mode-hopping events.

\section{THE USED APPROACH}

A considered configuration of a microcavity Brillouin laser under continuous pumping is shown in Fig.1. The cavity is coupled with the pump laser that excites a forward pump optical wave. Brillouin process is thought as an interaction between the forward pump wave $A_{F}(t)$ and the backward Stokes wave $A_{B}(t)$ through a sound wave $\rho(t)[27]$ :

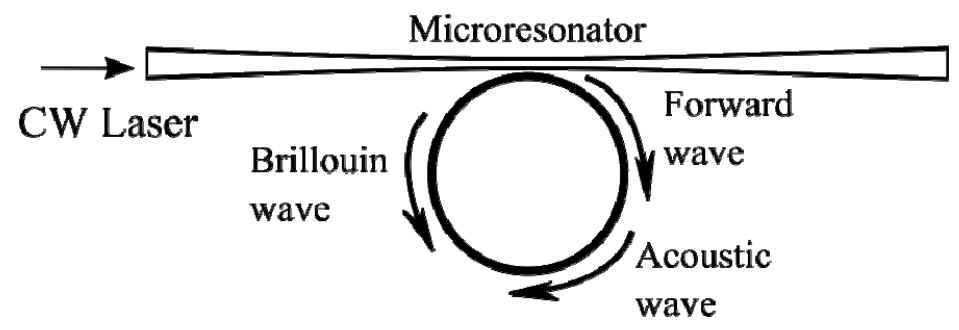

Figure 1. Brillouin lasing in a microcavity

$$
\begin{aligned}
& \frac{\partial A_{F}}{\partial t}=-\frac{A_{F}}{2 \tau_{F}}-i \frac{\gamma_{e} \omega_{F}}{4 n_{0}^{2} \rho_{0}} \Lambda_{F} A_{B} \rho+\sqrt{\frac{1}{\tau_{e x t}}} S \exp \left(i\left(\omega_{S}-\omega_{F}\right) t\right) \\
& \frac{\partial A_{B}}{\partial t}=-\frac{A_{B}}{2 \tau_{B}}-i \frac{\gamma_{e} \omega_{B}}{4 n_{0}^{2} \rho_{0}} \Lambda_{B} A_{F} \rho^{*} \\
& \frac{\partial \rho}{\partial t}=i \frac{\Omega_{b}^{2}-\Omega^{2}}{2 \Omega} \rho-\frac{\Gamma}{2} \rho-i \frac{\varepsilon_{0} \gamma_{e}}{4 \Omega} \frac{\ell_{\rho}^{2}}{R^{2}} \Lambda_{\rho} A_{F} A_{B}^{*}
\end{aligned}
$$

where $A_{F}(t), A_{B}(t), \rho(t)$ are the complex amplitudes, $\omega_{F}, \omega_{B}, \Omega$ are the frequencies, $l_{F}, l_{B}, l_{\rho}$ are angular moments and $\Lambda_{F}, \Lambda_{B}, \Lambda_{\rho}$ are the mode overlapping integrals for the pump, Stokes and sound waves, respectively. $\Omega_{b}$ is the Brillouin frequency shift, $\gamma_{e}$ is the electrostriction, $n_{0}$ is the cavity refractive index. Transversely the fields of the forward and backward optical waves are fundamental modes. The radius of the mode trajectory is assumed to be equal to the radius of the resonator $R$. The phase matching conditions are: $\Omega=\omega_{F}-\omega_{B}$ and $\ell_{\rho}=\ell_{F}+\ell_{B}$. For simplicity we set $\Lambda_{F}=\Lambda_{B}=\Lambda_{\rho}=1 / 2$ [27].

The time constants $\tau_{F}$ and $\tau_{B}$ are lifetimes for the forward and backward optical waves describing losses in the cavity. These losses can be accounted as the material losses and the losses due to coupling with the output fiber, i.e. for the forward and backward waves:

$$
\frac{1}{\tau_{F}}=\frac{1}{\tau_{F, 0}}+\frac{1}{\tau_{e x t}},
$$




$$
\frac{1}{\tau_{B}}=\frac{1}{\tau_{B, 0}}+\frac{1}{\tau_{e x t}}
$$

$S$ is the pump amplitude, $|S|^{2}$ is the pump power, $\left|A_{F}\right|^{2}$ and $\left|A_{B}\right|^{2}$ are energies of the forward and backward waves in the cavity [28]. They are linked as:

$$
\frac{\left|A_{B}\right|^{2}}{\tau_{B}}+\frac{\left|A_{F}\right|^{2}}{\tau_{0, F}}=|S|^{2}-|T|^{2},
$$

were $|T|^{2}-$ is the optical power transmitted through the cavity.

In the case of the pump wave frequency offset $\sigma_{F}=\omega_{S}-\omega_{F}$ the backward wave and sound wave frequencies are also detuned as $\sigma_{B}=\omega_{B}^{\prime}-\omega_{B}, \sigma_{\rho}=\Omega^{\prime}-\Omega$. New phase-matching is achieved as $\sigma_{F}=\sigma_{B}+\sigma_{\rho}$. New complex amplitudes could be expressed as $A_{F}^{\prime}=\left|A_{F}^{\prime}\right| \exp \left(i \varphi_{F}\right), A_{B}^{\prime}=\left|A_{B}^{\prime}\right| \exp \left(i \varphi_{B}\right), \rho^{\prime}=\left|\rho^{\prime}\right| \exp \left(i \varphi_{\rho}\right)$. Thus Eq. (1) is replaced by:

$$
\begin{aligned}
& \frac{\partial\left|A_{F}\right|}{\partial t}=-\frac{\left|A_{F}\right|}{2 \tau_{F}}+\frac{\gamma_{e} \omega_{0}}{8 n_{0}^{2} \rho_{0}}\left|A_{B}\right||\rho| \sin \left(\varphi_{\rho}+\varphi_{B}-\varphi_{F}\right)+\sqrt{\frac{1}{\tau_{\text {ext }}}}|S| \cos \left(\varphi_{S}-\varphi_{F}\right) \\
& \frac{\partial \varphi_{F}}{\partial t}=-\sigma_{F}-\frac{\gamma_{e} \omega_{0}}{8 n_{0}^{2} \rho_{0}} \frac{\left|A_{B}\right||\rho|}{\left|A_{F}\right|} \cos \left(\varphi_{\rho}+\varphi_{B}-\varphi_{F}\right)+\sqrt{\frac{1}{\tau_{e x t}}} \frac{|S|}{\left|A_{F}\right|} \sin \left(\varphi_{S}-\varphi_{F}\right) \\
& \frac{\partial\left|A_{B}\right|}{\partial t}=-\frac{\left|A_{B}\right|}{2 \tau_{B}}+\frac{\gamma_{e} \omega_{0}}{8 n_{0}^{2} \rho_{0}}\left|A_{F}\right||\rho| \sin \left(\varphi_{F}-\varphi_{B}-\varphi_{\rho}\right) \\
& \frac{\partial \varphi_{B}}{\partial t}=-\sigma_{B}-\frac{\gamma_{e} \omega_{0}}{8 n_{0}^{2} \rho_{0}} \frac{\left|A_{F}\right||\rho|}{\left|A_{B}\right|} \cos \left(\varphi_{F}-\varphi_{B}-\varphi_{\rho}\right) \\
& \frac{\partial|\rho|}{\partial t}=-\frac{\Gamma}{2}|\rho|+\frac{\varepsilon_{0} \gamma_{e}}{8 \Omega} \frac{\ell_{\rho}^{2}}{R^{2}}\left|A_{F}\right|\left|A_{B}\right| \sin \left(\varphi_{F}-\varphi_{B}-\varphi_{\rho}\right) \\
& \frac{\partial \varphi_{\rho}}{\partial t}=-\sigma_{\rho}+\frac{\Omega_{b}^{2}-\Omega^{2}}{2 \Omega}-\frac{\varepsilon_{0} \gamma_{e}}{8 \Omega} \frac{\ell_{\rho}^{2}}{R^{2}} \frac{\left|A_{F}\right|\left|A_{B}\right|}{|\rho|} \cos \left(\varphi_{F}-\varphi_{B}-\varphi_{\rho}\right) .
\end{aligned}
$$

where, primes are omitted and $\omega_{B} \approx \omega_{F}=\omega_{0}$. The steady-state solutions of Eq.3 are

$$
\begin{aligned}
\left|A_{F}\right|^{2} & =\frac{16 n_{0}^{2} \rho_{0} \Gamma \Omega R^{2}}{\tau_{B} \varepsilon_{0} \gamma_{e}^{2} \omega_{0} \ell_{\rho}^{2}} \frac{1}{\sin ^{2}\left(\varphi_{F}-\varphi_{B}-\varphi_{\rho}\right)} \\
\left|A_{B}\right|^{2} & =\frac{2 \tau_{B}}{\sqrt{\tau_{e x t}}}|S|\left|A_{F}\right| \cos \left(\varphi_{S}-\varphi_{F}\right)-\frac{\tau_{B}}{\tau_{F}}\left|A_{F}\right|^{2} .
\end{aligned}
$$

One can see that in steady-state, the energy of the forward wave does not depend on the pump power. It is determined by the cavity parameters and by the phase mismatch of the interacting waves. The second equation in Eq.4 expresses the energy conservation law (2), since $A_{F} / \sqrt{\tau_{\text {ext }}}=S+T$ [28]. Eq.(2) describes the link between the pump and Brillouin wave powers: 


$$
\frac{\left|A_{B}\right|^{2}}{\tau_{\text {ext }}}=\frac{\tau_{B}}{\tau_{\text {ext }}}\left(|S|^{2}-|T|^{2}-\frac{\left|A_{F}\right|^{2}}{\tau_{0, F}}\right) .
$$

Here, the parameters $\tau_{B} / \tau_{\text {ext }}$ and $\left|A_{F}^{\prime}\right|^{2} / \tau_{0, F}$ are responsible for the generation efficiency and threshold power, respectively

Let us now consider the generation parameters in the non-resonant case. The dependences of the optical energies on the frequency offset $\sigma_{F}$ could be obtained from Eq.3 and the phase matching conditions:

$$
\begin{gathered}
\sigma_{B}=\frac{\sigma_{F}-\left(\left(\Omega_{b}^{2}-\Omega^{2}\right) / 2 \Omega\right)}{1+\Gamma \tau_{B}} \\
\sigma_{\rho}=\frac{\sigma_{F} \Gamma \tau_{B}+\left(\left(\Omega_{b}^{2}-\Omega^{2}\right) / 2 \Omega\right)}{1+\Gamma \tau_{B}},
\end{gathered}
$$

The detuning $\sigma_{F}$ and the difference between the Brillouin shift and the inter-mode spacing $\Omega_{b}-\Omega$ determine the Brillouin laser frequency. The frequency offsets in their turn affect the phase mismatch of the interacting waves. Indeed, in the case of $\Omega_{b}=\Omega$, zero pump wave detuning requires $\sigma_{B}=\sigma_{\rho}=0$ and hence the steadystate phase matching $\varphi_{F}-\varphi_{B}-\varphi_{\rho}=\pi / 2$ and $\varphi_{S}-\varphi_{F}=0$. In the case of any detuning, the phase matching point is shifted leading to a change of the steady state wave energy. In particular, Eq.3 gives:

$$
\sin ^{2}\left(\varphi_{F}-\varphi_{B}-\varphi_{\rho}\right)=\frac{1}{1+4 \tau_{B}^{2} \sigma_{B}^{2}}
$$

leading to the implicit and explicit expressions for the forward and backward wave energies as functions of the frequency offset:

$$
\begin{aligned}
& \left|A_{F}\right|^{2}=\frac{g}{\tau_{B}}\left(1+\left(\frac{2\left(\sigma_{F}-\Delta\right) \tau_{B}}{1+\Gamma \tau_{B}}\right)^{2}\right), \\
& \left|A_{B}\right|^{2}=\sqrt{\frac{4 g \tau_{B}}{\tau_{e x t}}\left(1+\left(2 \sigma_{B} \tau_{B}\right)^{2}\right)} S \cos \left(\varphi_{s}-\varphi_{F}\right)-\frac{\tau_{B}}{\tau_{F}}\left|A_{F}\right|^{2}, \\
& \cos \left(\varphi_{s}-\varphi_{F}\right)=\left|\frac{\sqrt{1+\left(2 \sigma_{B} \tau_{B}\right)^{2}}}{2 \tau_{F}}+\frac{\frac{\left|A_{B}\right|^{2}}{2 g}}{\sqrt{1+\left(2 \sigma_{B} \tau_{B}\right)^{2}}}\right| \times \\
& \times\left(\left(1+\left(2 \sigma_{B} \tau_{B}\right)^{2}\right)\left(\sigma_{F}^{2}+\left(\frac{1}{2 \tau_{F}}\right)^{2}\right)+\frac{\left|A_{B}\right|^{2}}{2 g}\left(\frac{1}{\tau_{F}}-4 \sigma_{F} \sigma_{B} \tau_{B}\right)+\frac{\left|A_{B}\right|^{4}}{4 g^{2}}\right)^{-1 / 2},
\end{aligned}
$$

Here, $g=16 \rho_{0} \Gamma \Omega n_{0}^{2} R^{2} / \omega_{0} \varepsilon_{0} \gamma_{e}^{2} \ell^{2}, \Delta=\left(\Omega_{b}^{2}-\Omega^{2}\right) / 2 \Omega$. 
To analyze the obtained relations let us consider Brillouin lasing in silica disk (or sphere) cavity with a radius of $22 \mathrm{~mm}$ and mode area of $25 \mu \mathrm{m}^{2}$ [27]. We consider pumping at $\lambda=1550 \mathrm{~nm}\left(\omega_{0}=1.22 \cdot 10^{15}\right)$, the pump power of $1 \mathrm{~mW}\left(|S|^{2}=2.3 \cdot 10^{20} \mathrm{~W} / \mathrm{m}^{2}\right)$. Other system parameters are listed in Table 1 .

\section{Table 1}

\begin{tabular}{|l|l|l|l|}
\hline Parameter & Value & Parameter & Value \\
\hline$\tau_{F}=\tau_{B}$ & $\left(2 \pi \cdot 2 \cdot 10^{6}\right)^{-1} s$ & $\gamma_{e}$ & 1.5 \\
\hline$\tau_{\text {ext }}$ & $\left(2 \pi \cdot 1.33 \cdot 10^{6}\right)^{-1} s$ & $n_{0}$ & 1.5 \\
\hline$\Gamma$ & $\left(2 \pi \cdot 15.64 \cdot 10^{6}\right) s^{-1}$ & $\rho_{0}$ & $2200 \mathrm{~kg} / \mathrm{m}^{3}$ \\
\hline$\Omega_{b}$ & $\left(2 \pi \cdot 11.55 \cdot 10^{9}\right) s^{-1}$ & $\ell / R$ & $1.22 \cdot 10^{7} \mathrm{~m}^{-1}$ \\
\hline
\end{tabular}

In the first case the Brillouin shift is exactly equal to the spectral range between two microcavity modes $\Delta=0$. Fig. 2 (a) shows the dependencies of forward and backward waves energies on the detuning $\sigma_{F}$. With an increase of $\sigma_{F}$ the energy of the forward wave increases parabolically and symmetrically with respect to the change of $\sigma_{F}$ sign. In accordance with Eq.4 the forward wave energy does not depend on the pump power. At low pump powers, the threshold values of the forward wave energy are minimal at zero detuning $\sigma_{F}$. Generation of the backward wave occurs in a narrow spectrum range $\sigma_{B} \rightarrow 0$. It is important for practical realizations of narrow-band generation that the band of possible changes of $\sigma_{B}$ in $1+\Gamma \tau_{B}$ narrower than the band between the threshold points of $\sigma_{F}$ (see Eq.6). With a pump power increase, both the energy and available spectrum range of the Brillouin wave increase. The bandwidth between the threshold points also increases. Note that the dependence of the backward Brillouin wave energy on the frequency detuning is rather flat. 

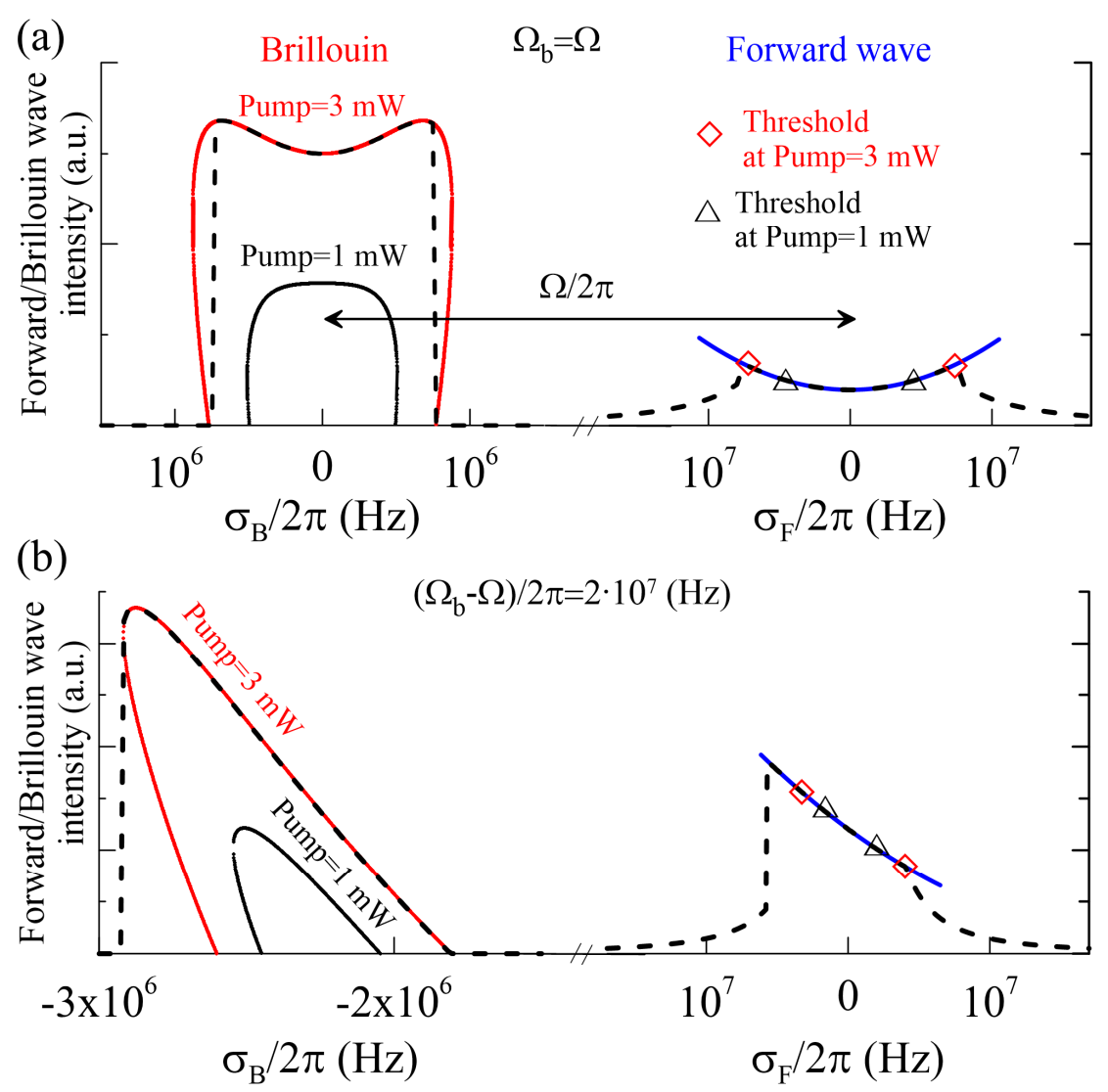

Figure 2. (a) The steady-state Brillouin wave energy as function of the detuning $\sigma_{B}$ for different pump powers (left) and the forward wave energy as function of the detuning $\sigma_{F}$ (right) for the resonant case when the Brillouin frequency shift coincides the cavity intermode spacing. (b) The same, but for non-resonant case when the Brillouin shift is detuned from the cavity intermode spacing by $\left(\Omega_{b}-\Omega\right) / 2 \pi=20$. Symbols denote forward wave energy levels corresponding to the Brillouin threshold. The results of numerical simulation (Eq.3)(dushed), the pump power is $3 \mathrm{~mW}$. Different scales for $\sigma_{F}$ and $\sigma_{B}$.

Let us now compare the considered resonant case with the case when the Brillouin shift exceeds the cavity intermode spacing $\Omega_{b}>\Omega$ (Fig. 2 (b)). In non-resonant case the frequency pulling effect of Brillouin wave generation toward to the peak gain takes place [29]. One can see that the detuning $\Delta / 2 \pi=20 \mathrm{MHz}$ shifts the Brillouin generation band by 2.5-3 MHz. The dependence of the forward wave energy on $\sigma_{F}$ is not in symmetry anymore, the minimum of the parabola is shifted to the point $\sigma_{F}=\Delta$, and the wave energy increases. The available band for $\sigma_{F}$ narrows. Accordingly, the generation band for $\sigma_{B}$ narrows as well. There is a range where the dependence of energy $\left|A_{B}\right|^{2}$ on the detuning is double-valued. The lower branch corresponds to unstable solutions. Due to the frequency pulling effect the maximum of the backward Brillouin wave energy is localized in the band with minimal $\sigma_{B}$ values limited by the condition of $\cos \left(\varphi_{s}-\varphi_{F}\right)=1$. Importantly, there is a range $\Delta$, where at sufficiently high pump powers the maximum value of the backward wave energy exceeds the level obtained for the resonant case $\Delta=\sigma_{B}=\sigma_{F}=0$. In particular, one can see from Fig. 2 that at $\Delta / 2 \pi=20 \mathrm{MHz}$, the pumping with a power higher than $1 \mathrm{~mW}$ causes an increase of the Brillouin wave peak 
energy compared to the resonant case $\Delta=\sigma_{B}=\sigma_{F}=0$. Assuming that the detuning $\Delta$ is high enough for pulling to overtake the characteristic losses $\sigma_{B}>1 / \tau_{B}$, we can estimate the parameters at which the effect occurs.

$$
\frac{\left(2 \sigma_{B} \tau_{B}-1\right)}{2\left(\sigma_{B} \tau_{B}\right)^{2}} S>\frac{1}{\tau_{F}} \sqrt{\frac{g \tau_{e x t}}{\tau_{B}}}
$$

\section{NUMERICAL SIMULATIONS AND RESULTS}

In this section we present results of numerical simulation of Eq.3. To simulate generation of the Brillouin wave from a noise a term describing low thermal density fluctuations (Langevin noise source) has been added to the third equation in Eq.1. The Langevin source $f(t)$ has the form of "white" noise equally splitted between the real

$f_{r}$ and imaginary $f_{i}$ parts $\left\langle f_{r}(t) f_{r}^{*}\left(t^{\prime}\right)\right\rangle=C \delta\left(t^{\prime}-t\right)$, where $C=k T \rho_{0} \Gamma / V v^{2}, k$ is the Boltzmann constant, $V$ is the volume of the acoustic mode, $v$ is the speed of the sound [30]. In established lasing regime the noise term determines the amplitude and phase fluctuation in the interacting waves. Without noise the interacting waves are fully coherent. Dynamics of the interacting wave energies at various pump power levels are simulated with the use of 4-order Runge-Kutta algorithm from a zero initial conditions to the steady-state level.

First, we compare the simulation and analytical results. The simulation results with parameters corresponding to the theoretical calculations for a pump power of $3 \mathrm{~mW}$ are shown in Fig. 2. One can see that they coincide with the results obtained from Eq.7. The only difference are unstable branches of the backward wave energies. They are not reproduced by the simulation. The simulation results are extended to the range $\sigma_{F}$ where the generation of the backward wave does not occur (the forward wave energy is below the threshold).

Let us consider how the Brillouin lasing in micro-cavity changes with a change of the pump power. In the case of resonant pumping, $\Delta=0$ (Fig. 3 (a)). As the frequency detuning $\sigma_{F}$ is changed the transmittance of the microcavity $|T|^{2} /|S|^{2}$ could be defined from Eq.2. For small $S \rightarrow 0$, the transmission spectrum is the transmission spectrum of the micro-cavity determined by $\tau_{F, 0}$ and $\tau_{\text {ext }}$ (dotted line in Fig. 3 (a)). With an increase of the pump power and excitation of the Brilouin wave the detuning $\sigma_{B}$ is determined by the detuning $\sigma_{F}$ (Eq.6). One can see that the simulation results coincide with the analytical results. With the increase of the pump power the energy transfers to the backward wave. The transmission of the resonator increases and the energy of the forward wave in the band of Brillouin lasing does not depend on the pump power.

Fig.3 (b) shows the Brillouin output power as a function of the pump power. One can see that at zero detuning $\sigma_{F}$ the threshold generation power is minimal. It could be obtained from Eq.4 :

$$
\left|S_{0}\right|^{2}=\frac{g \tau_{e x t}}{4 \tau_{B} \tau_{F}^{2}}
$$

A decrease in the differential generation efficiency with an increase of the pump power is governed by Eq.5, since $|S|^{2}$ and $|T|^{2}$ increase simultaneously. In accordance with the analytical predictions, non-zero pump frequency detuning increases the lasing threshold. However, at some pump power level, the output Brillouin power at non-zero pump frequency detuning exceeds its value at $\sigma_{F}=0$. It can be also seen from Eq.7 (see Fig. 
2 (a)): the energy level $\left|A_{B}\right|^{2}$ corresponding to the threshold detuning values exceeds its value in the central part at $\sigma_{F}=0$.

Let us consider now a case when the Brillouin shift exceeds the cavity inter-mode spacing $\Omega_{b}>\Omega$ (Fig. 3 (c)). The frequency pulling causes an asymmetry of transmission curve. The energies of the forward and backward waves coincide with the analytical results based on Eq.7. At a fixed pump power level, a narrower range of detuning $\sigma_{F}$ is available for Brillouin lasing than in the case of $\Omega_{b}=\Omega$. The latter leads to narrowing of the corresponding range of Brillouin frequencies $\sigma_{B}$. These effects are explained by an increase of the lasing threshold that is not zero even for resonant pumping. With an increase of the detuning $\sigma_{F}$ the threshold becomes higher. However, the use of high-power pumping allows to get Brillouin lasing with a power much higher than in the resonance case $\Omega_{b}=\Omega$ (Fig. 3 (d)).
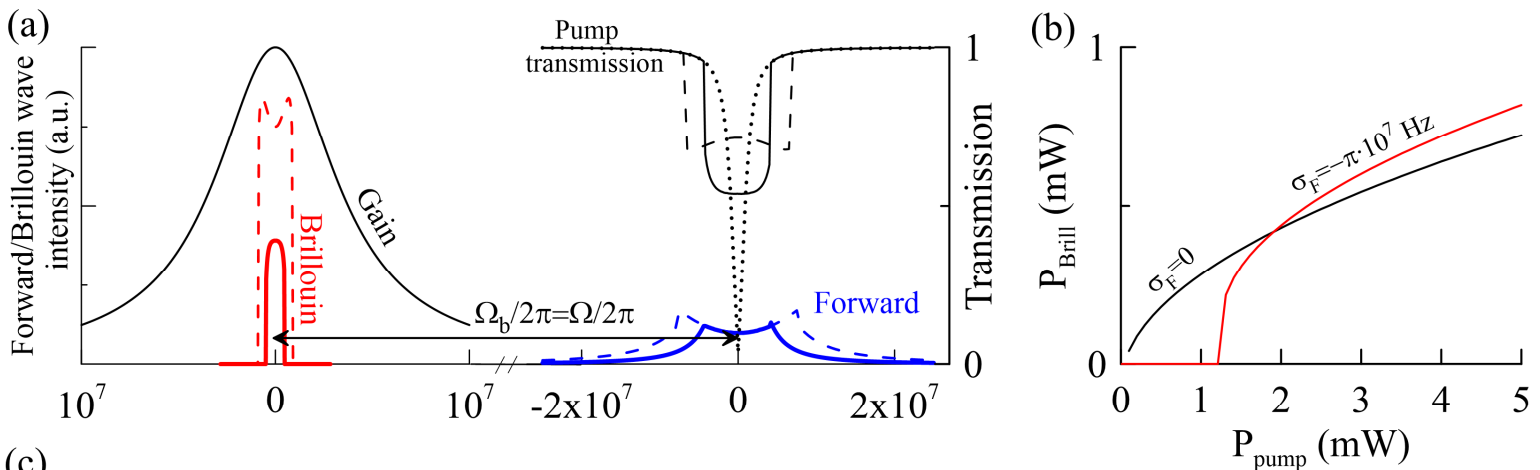

(c)
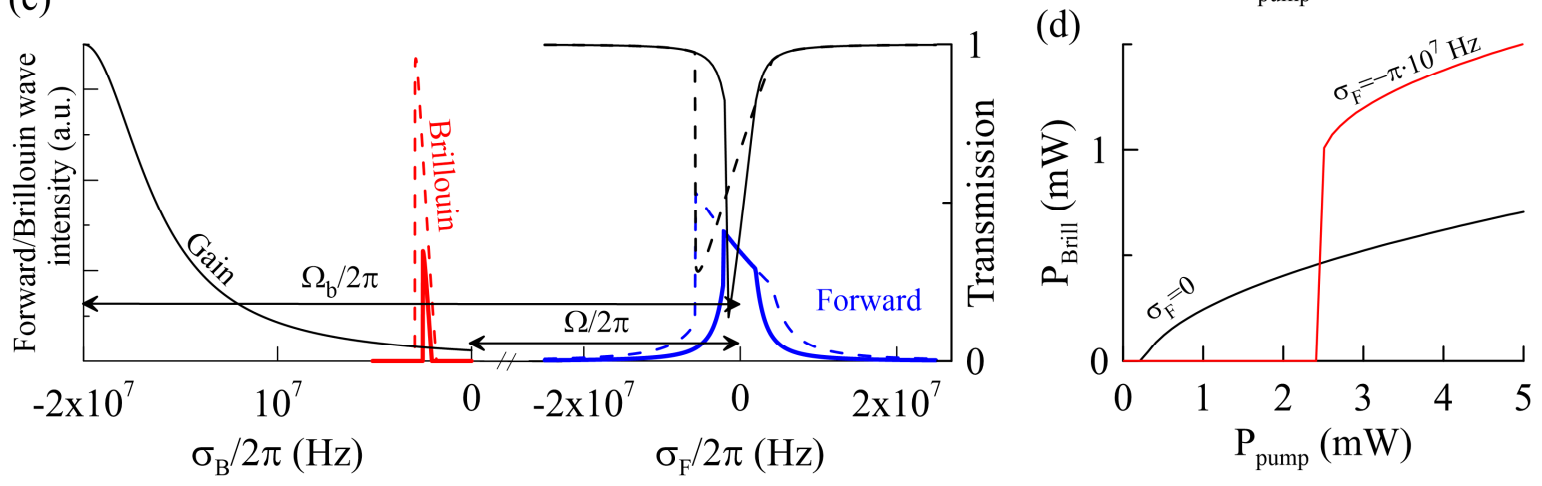

Figure 3. (a) The pump wave power introduced into the microcavity and the microcavity transmittance as functions as the pump frequency detuning $\sigma_{F}$ (right). The Brillouin wave intensity as a function of $\sigma_{B}$. Pump power is $1 \mathrm{~mW}$ (solid), $3 \mathrm{~mW}$ (dashed). The microcavity transmittance without Brillouin lasing (dotted). (b) The Brillouin laser power as a function of the pump power at various offsets $\sigma_{F}$. The spectral distance between the forward and backward waves coincides with the SBSshift $(a, b)$. The spectral distance between the forward and backward waves defers from the SBS frequency shift by $\left(\Omega_{b}-\Omega\right) / 2 \pi=20 \mathrm{MHz}(\mathrm{c}, \mathrm{d})$.

The reported features are also illustrated in Fig. 4. It shows the changes of the forward and backward wave energies depending on the corresponding frequency offsets at different values of $\Omega_{b}-\Omega$. The linear dependence of the detuning $\sigma_{B}$ on the value $\Omega_{b}-\Omega$ in agree with Eq.6. An increase of the forward wave intensity during the narrowing of the band $\sigma_{F}$ available for lasing and a jump of the forward wave intensity above the threshold 
are also in agreement with the previous observations. The figure illustrates the feature established above: in a certain range of detuning $\left(\Omega_{b}-\Omega\right)$ and at a sufficiently high pump power levels, the maximum value of the Brillouin wave energy is higher in comparison with its value in resonance. One can see that the pumping power of $1 \mathrm{~mW}$ is enough to increase the maximum energy of the backward wave in the range of detuning $0<\left(\Omega_{b}-\Omega\right) / 2 \pi \leq 30 \mathrm{MHz}$. The absolute maximum energy $\left|A_{B}\right|^{2}$ is fixed at $\left(\Omega_{b}-\Omega\right) / 2 \pi=20 \mathrm{MHz}$ and the pump detuning of $\sigma_{F} \approx 1.5 \mathrm{MHz}$. With a further increase of $\left(\Omega_{b}-\Omega\right)$ the energy of the Brillouin wave falls sharply. As the pump increases to $3 \mathrm{~mW}$, the Brillouin signal generation range increases significantly. In the whole considered range $0<\left(\Omega_{b}-\Omega\right) / 2 \pi \leq 50 \mathrm{MHz}$ the maximum of the Brillouin wave energy exceeds its value at the resonance. The absolute maximum of the Brillouin wave energy is observed at $\left(\Omega_{b}-\Omega\right) / 2 \pi=30 \mathrm{MHz}$ and a pump frequency detuning of $\sigma_{F} \approx 3 \mathrm{MHz}$.

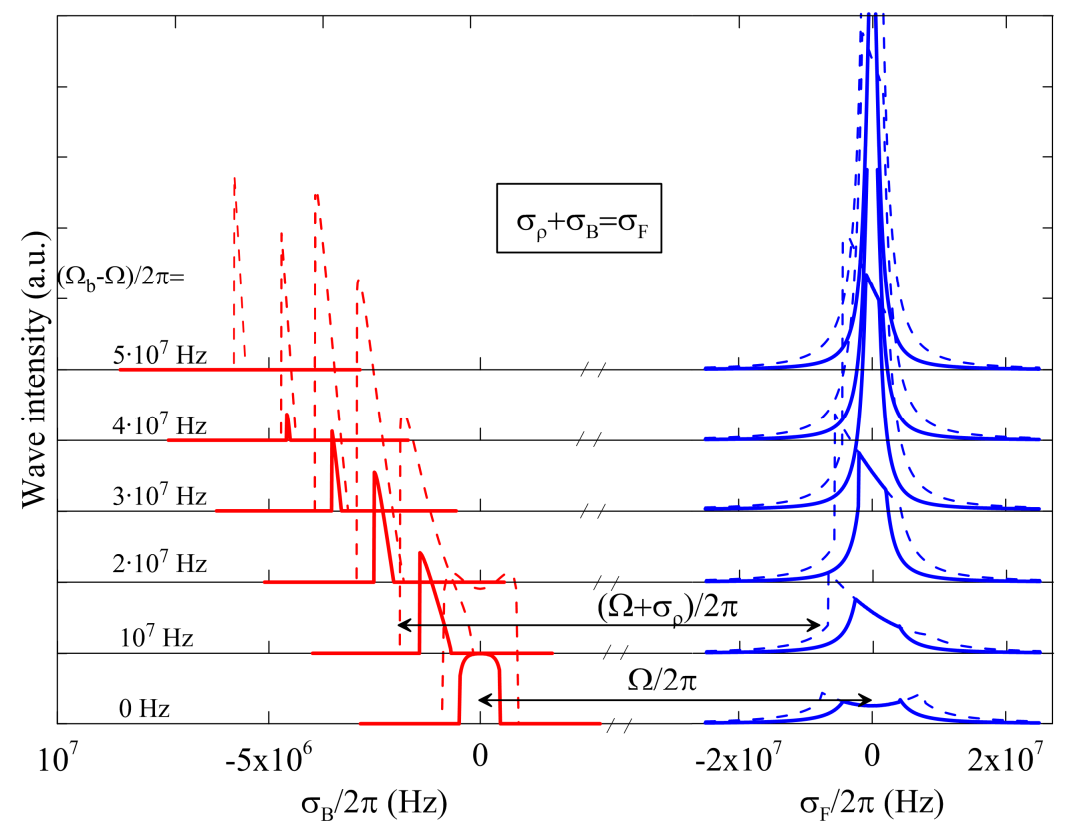

Figure 4. The intensities of the forward and backward waves as functions of the detuning $\sigma_{F}$ and $\sigma_{B}$, respectively, at different values of $\left(\Omega_{b}-\Omega\right) / 2 \pi$. The pump power is $1 \mathrm{~mW}$ (solid), $3 \mathrm{~mW}$ (dashed). Different scales are applied for $\sigma_{F}$ and $\sigma_{B}$ 
(a)

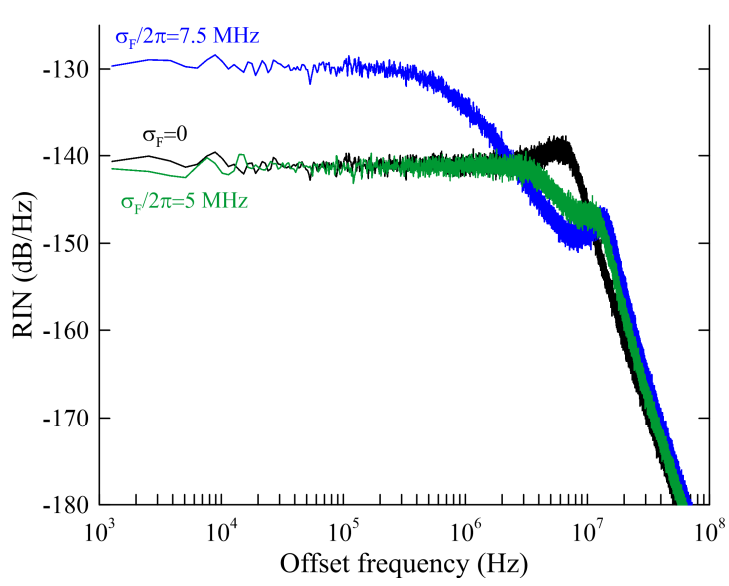

(b)

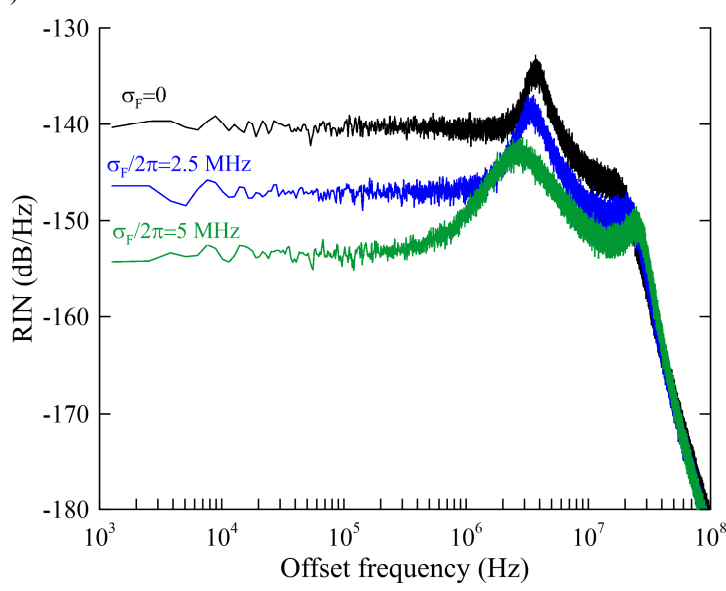

Figure 5. Results of numerical simulation. The relative noise intensity (RIN) spectral density for the Brillouin lasing in microcavity at different pump frequency detuning. $\left(\Omega_{b}-\Omega\right) / 2 \pi=0$ (a) and $\left(\Omega_{b}-\Omega\right) / 2 \pi=20$ (b).

The noise properties of the Brillouin lasing in the microcavity have been as well studied in the numerical experiment. The RIN (relative intensity noise) spectral density of the Brillouin radiation defined as

$$
S(\omega)=\frac{2}{\left\langle\left|A_{B}\right|^{2}\right\rangle^{2}} \int_{-\infty}^{+\infty}\left\langle\delta\left|A_{B}(t)\right|^{2} \delta\left|A_{B}(t+\tau)\right|^{2}\right\rangle \exp (i \omega \tau) d \tau
$$

has been evaluated for different values of the pump frequency detuning in two cases: $\left(\Omega_{b}-\Omega\right) / 2 \pi=0$ and $\left(\Omega_{b}-\Omega\right) / 2 \pi=20$. In the simulation, the pump power is $3 \mathrm{~mW}$. One can see that in the resonant case (Fig. 5 (a)) the dependence of the Brillouin intensity on the frequency detuning is flat. Therefore, a weak dependence of the spectral density RIN on the frequency detuning is observed up to $10 \mathrm{MHz}$. For $\sigma_{F} / 2 \pi \leq 5 \mathrm{MHz}$, the RIN is approximately $-140 \mathrm{~dB} / \mathrm{Hz}$. As the frequency detuning approaches the threshold values, RIN increases to $-130 \mathrm{~dB}$ within the band of $\sim 1 \mathrm{MHz}$.

As to concern RIN in a non-resonant case there is a remarkable result. There is an optimal pump frequency detuning for which the noise characteristics of the Brillouin lasing can be significantly improved (Fig. 5 (b)). One can see that at the detuning of $\sigma_{F} / 2 \pi=5 \mathrm{MHz}$ the RIN can be reduced down to $-155 \mathrm{~dB} / \mathrm{Hz}$. This effect is explained by narrowing of the Brillouin signal generation band and by suppression of its response to the phase and amplitude fluctuations of the interacting waves. Even despite a strong phase-amplitude coupling leading to formation of a characteristic noise maximum near 2-4 MHz, the RIN level remains lower than in the resonant case in a wide frequency band of $1 \mathrm{kHz}-50 \mathrm{MHz}$.

\section{CONCLUSION}


We have performed analytical and numerical treatments of the Brillouin lasing in microcavities. In addition to the previous works, we consider a non-resonant case of non-zero difference between the Brillouin shift and the microcavity inter-mode spacing. Despite an increase of the lasing threshold and shift of the gain maximum from the microcavity mode, the intensity of the Brillouin radiation can be higher than in the resonant case. A necessary condition for this effect is an optimized value of the pump frequency detuning. The result of the lasing threshold increase is the narrowing of the spectrum range when the Brillouin signal could be generated in the non-resonant case. Besides, with the optimized pump frequency detuning, the Brillouin laser noise is reduced. Analytical results are confirmed by numerical simulations. The results of the work could be important for design of microcavity Brillouin lasers, Brillouin sensors and other microwave devices of Brillouin photonics.

\section{Acknowledgments}

The work was supported by the Russian Science Foundation (grant №18-12-00457), Russian Foundation for Basic Research (grants №16-42-732135 R-OFIM, 18-42-732001 R-MK), Russian Ministry of High Education and Sciences (State contract 3.3889.2017/PCh). A. A. Fotiadi acknowledges a support from the Leverhulme Trust (Visiting Professor, Grant ref: VP2-2016-042).

\section{REFERENCES}

[1] Komljenovic, T., Srinivasan, S., Norberg, E., Davenport, M., Fish, G., \& Bowers, J. E. (2015). Widely tunable narrow-linewidth monolithically integrated external-cavity semiconductor lasers. IEEE Journal of Selected Topics in Quantum Electronics, 21(6), 214-222.

[2] Matei, D. G., Legero, T., Häfner, S., Grebing, C., Weyrich, R., Zhang, W., ... \& Sterr, U. (2017). 1.5 ì m Lasers with Sub-10 mHz Linewidth. Physical review letters, 118(26), 263202.

[3] Kobayashi, N., Sato, K., Namiwaka, M., Yamamoto, K., Watanabe, S., Kita, T. \& Yamazaki, H. (2015). Silicon photonic hybrid ring-filter external cavity wavelength tunable lasers. Journal of Lightwave Technology, 33(6), 1241-1246.

[4] C. J. Karlsson, F. A. Olsson, D. Letalick, and M. Harris. Appl.Opt. 39, 3716 (2000).

[5] Bueno Escobedo, J. L., Spirin, V. V., López-Mercado, C. A., Márquez Lucero, A., Mégret, P., Zolotovskii, I. O., Fotiadi, A. A., "Self-injection locking of the DFB laser through an external ring fiber cavity: Application for phase sensitive OTDR acoustic sensor," Results in Physics 7, 641-643 (2017).

[6] Hemmerich, A., McIntyre, D. H., Schropp Jr, D., Meschede, D., \& Hänsch, T. W. (1990). Optically stabilized narrow linewidth semiconductor laser for high resolution spectroscopy. Optics communications, 75(2), 118-122.

[7] E. IP, A. Lau, D. Barros, and J. Kahn, "Coherent detection in optical fiber systems," Opt. Express 16, 753791 (2008).

[8] M. Nakazawa, S. Okamoto, T. Omiya, K. Kasai, and M. Yoshida, "256-QAM (64 Gb/s) coherent optical transmission over $160 \mathrm{~km}$ with an optical bandwidth of $5.4 \mathrm{GHz}$," IEEE Photon. Technol. Lett. 22, 185-187 (2010).

[9] Yao, J. (2009). Microwave photonics. Journal of Lightwave Technology, 27(3), 314-335.

[10] Ohtsubo, J., [Semiconductor lasers: stability, instability and chaos], Springer Series in Optical Sciences, Springer Berlin Heidelberg (2013).

[11] K. Petermann, [Laser diode modulation and noise], Springer Science \&amp; Business Media (2012).

[12] Liang, W., Ilchenko, V. S., Eliyahu, D., Savchenkov, A. A., Matsko, A. B., Seidel, D., Maleki, L., "Ultralow noise miniature external cavity semiconductor laser," Nature Communications 6(1), 7371 (2015).

[13] W. Liang, V. S. Ilchenko, A. A. Savchenkov, A. B. Matsko, D. Seidel, and L. Maleki, "Whispering-gallerymode resonator-based ultranarrow linewidth external-cavity semiconductor laser," Opt. Lett. 35 2822-2824 (2010). 
[14] López-Mercado, C. A., Spirin, V. V., Bueno Escobedo, J. L., Márquez Lucero, A., Mégret, P., Zolotovskii, I. O.,Fotiadi, A. A., "Locking of the DFB laser through fiber optic resonator on different coupling regimes," Optics Communications 359, 195-199 (2016).

[15] Bueno Escobedo, J. L., Spirin, V. V., López-Mercado, C. A., Mégret, P., Zolotovskii, I. O., Fotiadi, A. A., "Selfinjection locking of the DFB laser through an external ring fiber cavity: Polarization behavior," Results in Physics 6, 59-60 (2016).

[16]Kobyakov, A., Sauer, M., \& Chowdhury, D. (2010). Stimulated Brillouin scattering in optical fibers. Advances in optics and photonics, 2(1), 1-59.

[17] Smith, S. P., Zarinetchi, F., \& Ezekiel, S. (1991). Narrow-linewidth stimulated Brillouin fiber laser and applications. Optics letters, 16(6), 393-395.

[18] Spirin, V. V., López-Mercado, C. A., Mégret, P., Fotiadi, A. A., "Single-mode Brillouin fiber laser passively stabilized at resonance frequency with self-injection locked pump laser," Laser Physics Letters 9(5), $377-$ 380 (2012).

[19] Grudinin, I. S., Matsko, A. B., \& Maleki, L. (2009). Brillouin lasing with a CaF 2 whispering gallery mode resonator. Physical review letters, 102(4), 043902.

[20]He, L., Özdemir, Ş. K., \& Yang, L. (2013). Whispering gallery microcavity lasers. Laser \& Photonics Reviews, 7(1), 60-82.

[21] Eggleton, B. J., Luther-Davies, B. \& Richardson, K. Chalcogenide photonics. Nat. Photonics 5, 141-148 (2011).

[22] Kabakova, I. V. et al. Narrow linewidth Brillouin laser based on chalcogenide photonic chip. Opt. Lett. 38, 3208-3211 (2013).

[23] Eggleton, B. J., Poulton, C. G., \& Pant, R. (2013). Inducing and harnessing stimulated Brillouin scattering in photonic integrated circuits. Advances in Optics and Photonics, 5(4), 536-587.

[24]H. Lee, T. Chen, J. Li, K. Y. Yang, S. Jeon, O. Painter, and K. J. Vahala, "Chemically etched ultrahigh-Q wedge-resonator on a silicon chip," Nat. Photonics 6, 369-373 (2012).

[25] Li, J., Lee, H., \& Vahala, K. J. (2013). Microwave synthesizer using an on-chip Brillouin oscillator. Nature communications, 4, 2097.

[26]Li, J., Suh, M. G., \& Vahala, K. (2017). Microresonator brillouin gyroscope. Optica, 4(3), 346-348.

[27] Loh, W., Papp, S. B., \& Diddams, S. A. (2015). Noise and dynamics of stimulated-Brillouin-scattering microresonator lasers. Physical Review A, 91(5), 053843.

[28] H. A. Haus, Waves and Fields in Optoelectronics (Prentice-Hall, 1983).

[29] Li, J., Lee, H., Chen, T., \& Vahala, K. J. (2012). Characterization of a high coherence, Brillouin microcavity laser on silicon. Optics express, 20(18), 20170-20180.

[30] Boyd, R. W., Rząewski, K., \& Narum, P. (1990). Noise initiation of stimulated Brillouin scattering. Physical Review A, 42(9), 5514. 